\title{
Profiling of gender-regulated gene transcripts in the filarial nematode Brugia malayi by cDNA oligonucleotide array analysis
}

\author{
Ben-Wen Li ${ }^{a}$,*, Amy C. Rush ${ }^{a}$, Seth D. Crosby ${ }^{b}$, Wesley C. Warren ${ }^{b}$, \\ Steven A. Williams ${ }^{\mathrm{c}}$, Makedonka Mitreva ${ }^{\mathrm{b}}$, Gary J. Weil ${ }^{\mathrm{a}}$ \\ ${ }^{a}$ Infectious Diseases Division, Washington University School of Medicine, Campus Box 8051, 660 S. Euclid Avenue, St. Louis, MO 63110, USA \\ ${ }^{\mathrm{b}}$ Genome Sequencing Center, Department of Genetics, Washington University School of Medicine, St. Louis, MO 63110, USA \\ ${ }^{\mathrm{c}}$ Department of Biological Science, Smith College, Northhampton, MA, USA
}

Received 23 December 2004; received in revised form 2 May 2005; accepted 11 May 2005

Available online 14 June 2005

\begin{abstract}
Microarray technology permits high-throughput comparisons of gene expression in different parasite stages or sexes and has been used widely. We report the first use of this technology for analysis of gene expression in filarial male and female worms. The slide array (comprised of 65-mer oligos representing 3569 EST clusters) was spotted with sequences selected from the extensive Brugia malayi EST database (http://zeldia.cap.ed.ac.uk/fgn/brugia.php). Arrays were hybridized with Cy dye labeled male and female cDNA. The experimental design included both biological and technical (dye-flip) replicates. The data were normalized for background and probe intensity, and the relative abundance of hybridized cDNA for each spot was determined. Genes showing two-fold or greater differences with $P<0.05$ were considered gender-regulated candidates. One thousand one hundred and seventy of 2443 clusters (48\%) with signals above threshold in at least one sex were considered as gender-regulated gene candidates. This included 520 and 650 clusters up-regulated in male and female worms, respectively. Fifty of 53 (94\%) gender-regulated candidate genes identified by microarray analysis were confirmed by real-time RT-PCR. Approximately $61 \%$ of gender-regulated genes had significant similarity to known genes in other organisms such as Caenorhabditis elegans. Many C. elegans homologues of these genes have been reported to have reproductive phenotypes (sterility or abnormal embryo development) by RNA interference. This study has provided the first broad view of gender-regulated gene expression in B. malayi; this should lead to improved understanding of reproduction in filarial nematodes. More generally, this approach holds great promise as a means of studying stage-specific or tissue-specific gene expression in parasitic nematodes.
\end{abstract}

(c) 2005 Elsevier B.V. All rights reserved.

Keywords: Microarray; Brugia; Filarial; Gene expression; Reproduction; Nematode

\section{Introduction}

Filarial nematodes cause important tropical diseases in humans such as onchocerciasis ("river blindness" caused by Onchocerca volvulus) and lymphatic filariasis ("elephantiasis" caused by Brugia malayi, B. timor and Wuchereria bancrofti). The parasites are dioecious, and they exhibit

Abbreviations: RT-PCR, reverse transcription polymerase chain reaction; RNA, ribonucleic acid; EST, expression sequence tag; MSP, major sperm protein; PMT, photomultiplier tube

* Corresponding author. Tel.: +1 314454 7782; fax: +1 3144545293.

E-mail address: bli@wustl.edu (B.-W. Li). marked sexual dimorphism. Brugia adult worms live in lymphatic vessels. The ovoviviparous females release microfilariae (MF) that are ingested by insects; these are essentially equivalent to the L1 stage of other nematodes. Microfilariae molt twice in competent insect vectors to become infective stage larvae (L3) that are infective to humans. L3 molt twice in the human host and to become adult worms that are reproductively active for years. Obviously, parasite reproduction is necessary for transmission, and transmission is necessary for reproduction. Improved understanding of the reproductive biology of filarial worms may provide new insights into the epidemiology of filariasis and lead to new tools for controlling these diseases. Basic research may identify new 
targets for intervention that interfere with egg, microfilaria, or sperm production [1,2]. Filariasis researchers stand to benefit from extensive functional genomic studies that have been performed on the free-living nematode Caenorhabditis elegans [3,4]. Since filarial parasites share many genes with $C$. elegans [5], information available on genes involved in reproduction in C. elegans is an important starting point for parallel studies in filarial worms [6]. However, there are important differences between filarial worms and C. elegans. For instance, while adult filarial worms exist as males (XY) and females (XX), C. elegans has males and hermaphrodites but no true females and no $\mathrm{Y}$ chromosome.

The large expressed sequence tag (EST) database and extensive genomic sequence information available for $B$. malayi [7-9] provide a solid foundation for studying the molecular biology of this organism using gene expression profiling and functional genomics approaches [10-13]. We have previously identified a small number of sex-regulated genes in B. malayi worms by differential display RT-PCR and real-time RT-PCR [11,12]. One of these genes (microfilaria sheath protein) was recently shown to be essential for normal production of microfilaria by RNA interference (RNAi) [13]. However, the pace of progress with this "molecule by molecule" approach is limited.

Microarrays can be used to rapidly assess and quantitate relative levels of expression of thousands of genes in parallel $[14,15]$. Early experience with small-scale arrays has demonstrated the potential value of this approach for studying gene expression in parasites [16-19]. We now report production and use of the first large-scale oligonucleotide microarray for a nematode parasite. We chose to first evaluate the array with a study of gender-linked expression because of prior work on this area in B. malayi and in C. elegans. Our results show that oligonucleotide microarray analysis is a reproducible, rapid, and highly efficient method for profiling gender-associated gene expression in B. malayi.

\section{Materials and methods}

\subsection{Parasite materials}

Adult B. malayi worms were isolated from the peritoneal cavity of infected jirds (Meriones unguiculatus) obtained from the NIAID Filariasis Repository (University of Georgia, Athens, GA). Male and female worms were carefully separated by size; broken worms were discarded. The worms were washed and immediately frozen at $-80^{\circ} \mathrm{C}$.

\subsection{RNA isolation and probe preparation}

Worms (usually 30 female and 100 male adult worms per batch) were crushed under liquid nitrogen with a ceramic mortar and pestle and extracted in TRIzol reagent (Invitrogen, Carlsbad, CA) as previously described [12]. The accuracy of worm gender separation was assessed by RT-PCR with $B$. malayi major sperm protein primers (BmMSP) and B. malayi embryo-associated fatty acid-binding protein primers $(\mathrm{Bm}$ $F A B-1)$ [11]. RNA quality was assessed with a model 2100 Bioanalyzer (Agilent, Palo Alto, CA). cDNA was synthesized from 5 to $7 \mu \mathrm{g}$ each of male and female total RNA samples using 3DNA capture sequence primers (3DNA Array 350 Detection system, Genisphere, Hatfield, PA) and SuperScript II Reverse Transcriptase (Gibco BRL, Gaithersburg, MD) for each probe according to standard protocols. cDNA was concentrated by Microcon YM-100 filter (Millipore) and either used immediately or stored at $-80^{\circ} \mathrm{C}$. cDNA was synthesized from two different male and female RNA samples (independently prepared as biological replicates).

A two-step protocol was used for hybridization (3DNA Array 350 Detection system, Genisphere, Hatfield, PA). First, oligo arrays were hybridized to the cDNA probes in $2 \times$ SDS based-hybridization buffer and washed in $2 \times \mathrm{SSC}, 0.2 \%$ SDS according to the manufacturer's protocol. Fluorescent $\mathrm{Cy} 3-$ and Cy5-capture reagents were combined in hybridization buffer and added to each array. The arrays were incubated and washed as above. Each experiment consisted of a pair-wise competitive hybridization of cDNA samples from female and male worms and a reciprocal dye-flip replicate. Since biological duplicates were tested, a total of four DNA microarrays were used for comparison of the two types of cDNA.

\subsection{Microarray fabrication}

B. malayi clusters for arrays were selected from 8392 clusters generated by the Filarial Genome Project and posted at the website http://zeldia.cap.ed.ac.uk/fgn/brugia.php [7]. These clusters were derived from $15 \mathrm{cDNA}$ libraries that represent the major $B$. malayi life cycle stages. They represent approximately $40 \%$ of the total number of predicted genes for B. malayi [8]. Clusters with multiple ESTs, or with detectable similarity to proteins in public databases using BLAST [20], and sequence permitting design of a unique 65-mer oligonucleotide were chosen for inclusion on the array. Oligonucleotides were synthesized from the consensus sequence of selected clusters $(n=3569)$ by standard methods by Illumina (San Diego, CA). The oligonucleotides ( $50 \mathrm{nM}$ in $3 \times$ SSC with $0.75 \mathrm{M}$ betaine) were printed in duplicate on MWG Epoxy slides (MWG Bioteche Inc, High Point, NC) by a locally constructed linear servo arrayer (after the DeRisi model, http://derisilabs.ucsf.edu/).

\subsection{Data processing and analysis}

Slides were scanned immediately after hybridization on a ScanArray Express HT Scanner (Perkin-Elmer, Boston, MA) to detect Cy 3 and Cy5 fluorescence at 543 and $633 \mathrm{~nm}$, respectively. Laser power was kept constant for $\mathrm{Cy} 3 / \mathrm{Cy} 5$ scans, and photomultiplier tube values were 69 and $60 \mathrm{~V}$, respectively. An additional scan was done for each slide with the PMT set for 54 and $46 \mathrm{~V}$. The high PMT scan was done in order to maximize signal from low intensity spots. Likewise, 
the low PMT scan was done in order to characterize differential hybridization for high intensity spots which appeared saturated in the high PMT scan. Griding and analysis of images was performed with ScanArray software Express V2.0 (Perkin-Elmer, Boston, MA). Each spot was defined on a pixel-by-pixel basis, using a modified Mann-Whitney statistical test. The resultant values were background subtracted and Lowess [21] normalized by using GeneSpring 6.1 software (Silicon Genetics, Redwood City, CA). Twenty percent of the data were used to calculate the Lowess fit at each point. Oligonucleotide elements that received "present" (intensity > 200 intensity unit or the signal/background > 2) calls in all four microarrays and displayed $>700$ or $>127$ background subtracted intensity units (high or low PMT settings, respectively) in two of four channels for either $\mathrm{Cy} 3$ or Cy5 were identified, and all others were excluded from the analysis. The $\log _{2}$ ratio of median dye intensities for each remaining element was averaged across all four microarrays. Data were filtered using the Student's $T$-test function in GeneSpring. Genes with differences with $P$-values $<0.05$ in either the high or the low PMT scans and that had arithmetic ratios $\geq 2.0$ were considered to be significantly genderregulated. The best $P$-value obtained with high or low PMT scans and its accompanying arithmetic ratio were reported. Multiple testing corrections were not used in order to minimize type- 2 error.

\subsection{Real-time RT-PCR to confirm selected gender-regulated candidate genes identified by microarray analysis}

Selected gender-regulated gene candidates from the microarray analysis were chosen for confirmation studies by real-time RT-PCR as previously described in detail [12]. Briefly, complementary DNA primers (primer sequence information is available upon request) were designed from EST sequences obtained from GenBank with Primer Express software (Version 1.0, PE Applied Biosystems, Foster City, CA). The PCR reactions were carried out in 96 well microtiter plate wells in a $25 \mu \mathrm{l}$ reaction volume with SYBR Green Master Mix (PE Biosystems, Forster City, CA) with optimized concentrations of specific primers. Experimental design and relative quantification calculations were carried out according to RQ software (Relative Quantification (RQ) ABI Prism 7000 Sequence Detection System). Nicotinamide adenine dinucleotide dehydrogenase subunit 1 (BMC02280, NADH subunit 1) was used as a not gender-regulated internal control [12].

\subsection{Homology search of B. malayi genes}

A similarity search was performed using WU-BLASTX and WU-TBLASTX (https://blast.wustl.edu); [20] with 1170 gender-regulated cluster consensus sequences as queries versus multiple databases including $C$. elegans proteome (Wormpep version 132) (Wellcome Trust Sanger Institute, unpublished), non-redundant protein GenBank (11/14/04) [22] and nematode nucleotide sequences [23] with B. malayi ESTs removed. Homologues were reported for $E$-values less than or equal to $1 \mathrm{e}-20$.

To identify cases in which $C$. elegans homologues of $B$. malayi clusters have been surveyed for phenotypes in $C$. elegans using RNAi, the clusters with significant matches to $C$. elegans proteins were cross-referenced to a list of $17913 C$. elegans genes for which information on RNAi-induced phenotypes was available (http://www.wormbase.org/) [24,25]. All matches with similarities with $E$-values less than or equal to $1 \mathrm{e}-20$ were reported.

\section{Results}

\subsection{Gender-regulated gene transcripts were reproducibly detected by oligo arrays}

As our study represents the first use of oligonucleotide microarrays to study gene expression in B. malayi, we took great care to ensure the validity of our results. Our first objective was to ensure that similar levels of high quality total RNA was isolated from each sexually mature parasite population. Bioanalyzer 2100 results showed that all RNA samples isolated from male and female worms had equivalent quality and displayed minimal degradation (data not shown). Additional evidence that equivalent quantities of male and female total RNA were used for each hybridization experiment was obtained by examining relative expression levels of internal controls [NADH subunit 1 (BMC02280) and histone H3 (BMC00764)] previously shown to be expressed equally in both sexes by real-time RT-PCR [12]. Expression of these control genes was equal in male and female worms by microarray analysis.

\subsection{Expression profiling}

A complete list of clusters for the arrays, oligonucleotide sequences, and hybridization data is posted at the NCBI GEO database [26] http://www.ncbi.nlm.nih.gov/ projects/geo/query/acc.cgi?acc=GPL1483. Of 3569 clusters represented on the array, 2443 had hybridization signals above the threshold for at least one sex. One thousand one hundred and seventy (48\% of these clusters and $33 \%$ of total clusters on the microarray) met our criteria for gender-regulated expression (two-fold or greater differences and $P$-values <0.05) (Fig. 1). Of 1170 clusters, 455 clusters $(39 \%)$ had no significant similarity to known proteins in other organisms. This is somewhat higher than the percentage of novel genes represented on the array (1160 of 3569 clusters or $32.5 \%$ ). The differentially expressed clusters included 520 and 650 clusters up-regulated in male and female worms, respectively. The frequency distribution of higher:lower expression ratios is shown in Fig. 2. Although there were more female up-regulated gene candi- 


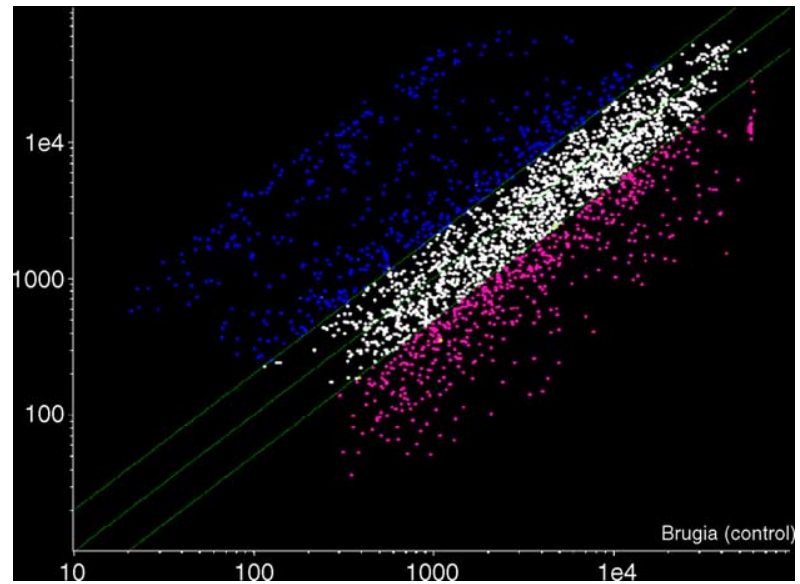

Fig. 1. Expression profiling of clusters from Brugia malayi. This is scatter plot of the mean normalized fluorescence intensity values from the male versus female comparison derived from the low PMT scan. Expression values that are $\geq 2$-fold (the flanking green lines) and $P<0.05$ in male are blue (upper left) and those that are $\geq 2$-fold higher and $P<0.05$ in females are dark pink (lower right). White spots are less than two-fold unregulated. Spots outside the two-fold line that are not red or blue have a $P>0.05$.

dates, expression ratios for male up-regulated genes tended to be higher. The median expression ratios for male and female up-regulated gene candidates were 3.88 and 3.01, respectively. The frequency of male up-regulated clusters with expression ratios of 10 -fold or greater was much higher (169 of 520 , or $32.5 \%$ ) than that in female-enriched clusters $(57$ of 650 , or $9 \%)\left(P<0.001\right.$ by $\left.\chi^{2}\right)$. Functional class information for male up-regulated and female up-regulated clusters with significant similarity to known genes is listed in Table 1. Functional classes for the most highly male or female up-regulated known genes (expression ratios $\geq 10$ ) in B. malayi are shown in Table 2.

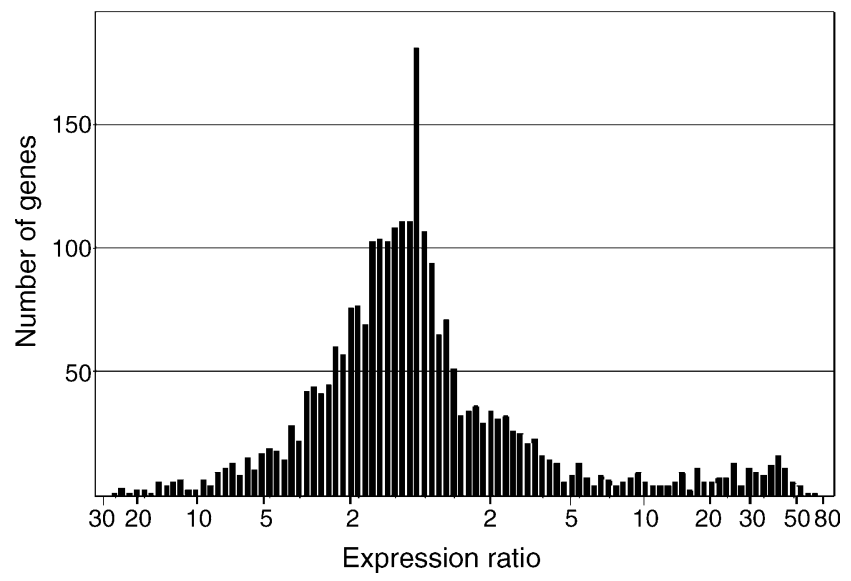

Fig. 2. Frequency distribution of expression ratios obtained from B. malayi clusters on a microarray probed with male- and female-specific cDNA. The signal intensity when probed with Cy5-labelled cDNA derived from female worms was divided by that of the Cy3-labelled cDNA derived from males or vice versa and then $\log _{2}$ transformed. Expression ratios were plotted on the abscissa and frequency was plotted on the ordinate.
Table 1

Number of gender-regulated clusters by functional class

\begin{tabular}{lcc}
\hline Description & Male & Female \\
\hline Cytoskeleton & 28 & 0 \\
Muscle & 5 & 2 \\
Major sperm protein & 8 & 0 \\
Oxidation-reduction & 11 & 0 \\
Protease & 4 & 0 \\
AvL3-1 & 4 & 0 \\
Zinc-finger proteins & 3 & 0 \\
DNA synthesis & 5 & 0 \\
NADH dehydrogenase subunit 5 & 4 & 0 \\
$\quad$ and 6 & & 27 \\
Protein synthesis & 6 & 15 \\
Phosphatase/kinase & 7 & 4 \\
ATP synthase & 0 & 8 \\
Citric acid cycle enzymes & 0 & 6 \\
Histones & 0 & 5 \\
Egg/embryo development & 0 & 4 \\
Brugia serine proteinase & 0 & 2 \\
$\quad$ inhibitors (Bm-spn-1 and 2) & & 5 \\
Chaperonin/heat shock protein & 0 & 6 \\
GTP-binding/Ras-related & 0 & 3 \\
Receptors (hormone, sperm and & & \\
$\quad$ nuclear) & 0 & \\
Ion channels & & \\
\hline & & \\
\hline
\end{tabular}

\subsection{Independent confirmation of gender-regulated genes identified by the oligo array with real-time RT-PCR}

To verify the gender-regulated genes identified by the arrays, we examined gene expression in adult male and female worms by real-time RT-PCR analysis. Fifty-five gender-regulated gene candidates identified by microarray analysis were selected for real time RT-PCR analysis. Twenty-eight of these genes were up-regulated in female

Table 2

Highly gender-regulated, known proteins in B. malayi adult worms

\begin{tabular}{llc}
\hline Description & Male & Female \\
\hline Major sperm protein & 12 & 0 \\
$\quad$ (MSP)/MSP-like & & \\
Serine/threonine kinase & 4 & 0 \\
Phosphatase & 4 & 0 \\
PDZ/LIM/PAZ-domain & 4 & 0 \\
Tyrosin protein & 2 & 0 \\
Protein phosphatase/kinase & 4 & 0 \\
Zinc-finger & 2 & 0 \\
Trehalase & 2 & 0 \\
Homolog of human & 2 & 0 \\
$\quad$ gonadotropin release & & \\
$\quad$ hormone receptor & & 4 \\
Sheath proteins & 0 & 1 \\
PAN-domain protein & 0 & 2 \\
Ion-channel & 0 & 2 \\
Nuclear receptor & 0 & 3 \\
Caveolin/High mobility & 0 & 8 \\
$\quad$ protein/Glutathione & & 20 \\
Others & 24 & \\
Total & 60 &
\end{tabular}

Expression ratio $>10$. 
Table 3

Real-time RT-PCR to confirm selected sex-regulated candidate genes identified by microarray analysis

\begin{tabular}{lllll}
\hline & $\begin{array}{l}\text { Identified } \\
\text { by array }\end{array}$ & $\begin{array}{l}\text { Confirmed by } \\
\text { real-time } \\
\text { RT-PCR }\end{array}$ & $\begin{array}{l}\text { Identified by } \\
\text { real-time } \\
\text { RTPCR }\end{array}$ & $\begin{array}{l}\text { Confirmed } \\
\text { by array }\end{array}$ \\
\hline Female & 27 & 27 & 9 & 9 \\
Male & 26 & 23 & 8 & 8 \\
Total & 53 & $50(94 \%)$ & 17 & $17(100 \%)$ \\
\hline
\end{tabular}

worms and 27 were up-regulated in males. Expression ratios for these genes ranged from 2- to 44 -fold by microarray analysis. Two genes gave no satisfactory results with the primers used. Fifty of the 53 remaining genes (94\%) were confirmed by real time RT-PCR to have gender-regulated expression in the same direction as was observed by microarray analysis (Table 3). We also examined microarray results for clusters previously identified as having gender-regulated expression by real-time RT-PCR [12]. All of the genes with gender-regulated expression by real-time RT-PCR were verified by microarray analysis (Table 3 ). A strong correlation was observed between fold difference values obtained by microarray analysis and log fold differences by real time RTPCR (Fig. 3).

\subsection{Male up-regulated clusters}

Of the 520 candidate male up-regulated clusters, 320 $(62 \%)$ had significant similarity to known genes in other organisms (Suppl. Table 1). Of these, 210 (66\%) had significant similarity to genes in C. elegans (Suppl. Table 1). One hundred and sixty-nine of these $(80 \%)$ have been studied by RNAi in C. elegans [24,25] (Suppl. Table 2), of which, 57 $(57 / 169 ; 34 \%)$ had a reproduction phenotype such as sterility.

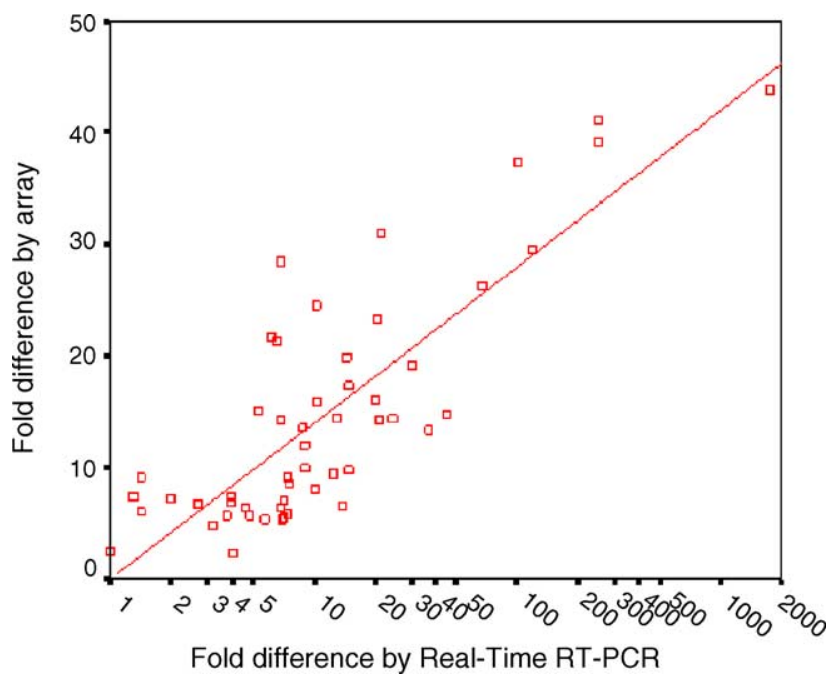

Fig. 3. This semi-log plot shows the relationship between gene expression values (expressed as fold-differences) obtained by microarray and by realtime RT-PCR for selected genes with gender-biased expression by microarray (Spearman rank correlation coefficient, $0.73, P<0.001$ ).
Male up-regulated clusters can be divided into four groups: (1) genes previously reported to be male up-regulated in filarial parasites, such as major sperm proteins (MSPs) [11,12]; (2) genes reported to be male up-regulated in other nematodes, such as a transcription factor (zinc finger, BMC07636 and BMC98240), a malate dehydrogenase (BMC04971), and a serine/threonine phosphatase (BMC10182) [18,27-29]; (3) genes that were newly identified as male up-regulated, such as genes with sequence similarity to muscle and cytoskeleton proteins (e.g. myosin, paramyosin, intermediate filament, troponin and actin) and genes related to cellular and extracuticular extracellular metabolism (e.g. cuticular glutathione peroxidase (BMC02584, BMC06710 and BMC12436), cytochrome oxidases (BMC04127)); (4) novel genes (38\% of the total). MSP proteins and MSP-like domain proteins, serine/threonine phosphatases and kinase were most abundant among highly male-biased expressed known genes (expression ratios $\geq 10$, Table 2 ); these accounted for 20 and $10 \%$ of the total, respectively. The B. malayi homologues (BMC06058 and BMC11125) of C. elegans gene coding for F54D7.3 protein (ortholog of the human gene gonadotropin release hormone receptor gene) were also highly up-regulated in males.

\subsection{Female up-regulated clusters}

Of the 650 candidate female-regulated clusters, 395 $(61 \%)$ encoded proteins with homologues in other organisms (Suppl. Table 3). Of these, 303 clusters (77\%) had a C. elegans homologue (Suppl. Table 3). Two hundred and thirty-seven of these genes $(237 / 303 ; 79 \%)$ have been studied by RNAi in C. elegans (Suppl. Table 2) [24,25], and $112(112 / 237 ; 47 \%)$ of them had phenotypes. Most phenotypes were related to embryogenesis or embryo development. Female up-regulated clusters with significant similarity to known genes fell into four groups: (1) genes previously confirmed as being female up-regulated including microfilarial sheath protein, high mobility protein, fatty acid binding protein and caveolin in filarial nematodes [12,13]; (2) genes previously reported to be female up-regulated in other nematodes. These included genes involved in transcription and translation (e.g. nuclear hormone receptors, RNA polymerase, tRNA synthetase, ribosomal proteins and elongation factor), protein processing/trafficking (heat shock proteins and chaperonin), and genes involved in embryonic development and growth in C. elegans (e.g. protein F52B11.3-BMC07645, BO250.1-BMC00336 and F33H1.4BMC09458); (3) genes newly identified as female-regulated including those involved in nematode reproduction (such as egg receptor for sperm, B. malayi serine proteinase inhibitor, collagen, multi-drug resistance factor, and tubulin); (4) novel genes (39\% of the total). Known genes with expression ratios $\geq 10$ are listed by functional class in Table 2 . Female upregulated genes identified previously such as sheath proteins, caveolin and high mobility protein were also highly female up-regulated in this study. 


\section{Discussion}

This paper presents the first use of oligonucleotide microarrays to profile gene expression in a filarial nematode. We focused on gender in this paper because of the relative ease of obtaining RNA from adult worms and the availability of pilot data on gender-biased genes from our prior work $[11,12]$. Our results show that gene expression profiles of $\sim 48 \%$ (1170/2443) of clusters that hybridized with cDNA from adult worms were gender-regulated. This is a higher percentage than that reported for C. elegans $(\sim 12 \%)$ [27] and lower than that reported for Trichostrongylus vitrinus (72\%) [18]. These differences may be due to selection bias of genes represented on the chips and may not reflect biological differences between these nematodes. The $C$. elegans microarray contained 17,871 genes and represented about $94 \%$ of the 18,967 genes annotated, while the T. vitrinus microarray contained only 716 ESTs from gender-selected cDNA libraries. There are also biological differences between these nematode species. For example, the uteri of mature Brugia female worms are often full of microfilariae, which are essentially equivalent to the $\mathrm{L} 1$ stage in C. elegans and T. vitrinus. Obviously, female up-regulated genes in B. malayi will include genes expressed by developing microfilariae.

Our experiments identified 520 male up-regulated genes and 650 female up-regulated genes with expression ratios $\geq 2$ that were significant at the $95 \%$ confidence level. Considering that females produce eggs and embryos, we expected to find many more female up-regulated clusters than male upregulated clusters. The finding that the mean expression ratio for male up-regulated clusters was higher than that for female up-regulated clusters was consistent with results reported for male up-regulated clusters in C. elegans [27].

Several lines of evidence support the validity of microarray results obtained in this study. First, reproducibility was good in biological replicate samples. Seventy-nine percent of the genes with gender-regulated expression in one biological sample also had gender-regulated expression in a second biological sample. Second, 94\% of selected genderregulated clusters identified by microarray were confirmed independently by real-time RT-PCR (Table 3). Third, all genes previously reported to have gender regulated expression by real-time RT-PCR [12] were confirmed to have gender regulated expression by microarray, and there was a strong correlation between fold-difference values obtained by these two methods. Fourth, many of the genes identified in our array experiment have reproductive phenotypes in C. elegans.

\subsection{Gene expression in males}

Thirty-eight percent $(199 / 520)$ of the male-regulated $B$. malayi clusters were novel. Forty percent (210/520) of the male-regulated clusters had a $C$. elegans homologue, and many of these have been reported to be male up-regulated in C. elegans when compared with hermaphrodites [27]. Genes involved in reproductive processes (e.g. major sperm proteins), molecules in energy storage and supply, cytoskeleton and muscle-related molecules, and enzymes in cellular metabolism especially in oxidation-reduction (cuticular glutathione peroxidase and cytochrome oxidase) were most notable amongst male up-regulated annotated clusters. The differential expression of these transcripts in male worms is likely to be related to their biological functions in reproduction.

Major sperm proteins (MSPs) are nematode-specific cytoskeleton proteins comprising $\sim 15 \%$ of total sperm protein $[30,31]$. These are among the most highly represented ESTs in B. malayi [8]. MSPs were also highly represented in male-enriched datasets for both T. vitrinus and C. elegans [3]. MSPs are involved in the movement of the amoeboid nematode sperm [32]. Recent studies show that MSPs also have a signaling role in oocyte production and maturation in C. elegans $[33,34]$. The retention of this MSP signaling function in Ascaris suum [34] suggests that this may also be present in filarial nematodes. Thus, the finding that $20 \%$ of highly male-up-regulated clusters in our study were either MSPs or homologues of C. elegans genes that contain an MSP domain is impressive but not surprising (Table 2).

In addition to MSPs, clusters with significant similarity to $C$. elegans serine/threonine kinases and phosphatases were also common among highly male up-regulated transcripts (Table 2). These genes are also highly expressed in C. elegans germ line tissue [3] and in the T. vitrinus male-selected cDNA library [18]. These enzymes might play important roles in regulating sperm maturation by posttranslational modification [3] and in signaling cascades or protein modification within the oocyte following fertilization [1,29]. Knockouts of the C. elegans homologue of a male-enriched serine/threonine protein phosphatase gene Od-mpp 1 from the parasitic nematode Oesophagostomum dentatum caused sterility of hermaphrodites $[1,29]$ through impaired sperm function [35]. The B. malayi homologue of this serine/threonine phosphatase (BMC10182) was highly up-regulated in males with expression ratios of 37 by microarray and 101 by real-time RT-PCR.

Several other groups of male up-regulated clusters contained special domains thought to be related to reproductive processes. For instance, B. malayi homologues of the C. elegans PDZ-domain (C25G4.6, pfam00595) (clusters BMC11959 and BMC04055) were highly male up-regulated (expression ratios $\geq 20$ ). This is consistent with the findings in the $T$. vitrinus male-selected cDNA library and in C. elegans microarray experiments $[18,27]$. The PDZ domain, a common modular protein-interaction domain, is believed to be involved in diverse signal transduction pathways [36]. RNAi of the $C$. elegans homologue with a PDZ domain (C25G4.6) caused sterility in the treated worms or their progeny, suggesting a role in gamete development [24,35]. In addition to the PDZ domain containing proteins, two other clusters highly expressed in B. malayi males had significant similarity to a PAZ domain (pfam02170) containing protein in C. elegans (ZK757.3B). This domain is found in the PIWI-domain gene 
family that is essential for producing and maintaining germline stem cells responsible for gametogenesis in Drosophila [37]. PIWI-domain gene family members are enriched in the C. elegans germline-intrisic group (genes expressed similarly in germ lines making only sperm or only oocytes) [3].

Several cytoskeleton and muscle-related molecules were male up-regulated with ratios ranging from 2 to 7 . These included 33 clusters with sequence significant similarity to myosin, paramyosin, troponin and actin (Table 1). Genes encoding tegument-associated molecules such as actin, tropomyosin and dynein light chain have previously been shown to be differentially expressed in adult Schistosoma mansoni males [38,39]. The explanation suggested for this was that males are larger (with more tegument and muscle) and more active in copulation than females [40,41]. However, this may not apply to B. malayi, since Brugia females seem to be as active as males in vitro, and there is no obvious difference in relative muscle content.

Another possible muscle-related male up-regulated $B$. malayi cluster (BMC01860) is a homologue of C. elegans gene Y1A5A.1, a male-enriched LIM domain (pfam00412) containing protein with a homologue in $T$. vitrinus $[3,18,27]$. Onchocerciasis patients and animals vaccinated with irradiated filarial L3 produce strong antibody responses to a related protein cloned from the filarial worm Acanthocheilonema viteae (AvL3-1) [42]. LIM domain-containing proteins are involved in diverse biological processes including cytoskeleton organization and cell lineage specification by mediating protein-protein interactions. The reported localization of OvL3-1 (the Onchocerca volvulus homologue of AvL3-1) in muscle tissue [43] suggests that this LIM domain protein may interact with muscle proteins.

Several genes encoding enzymes involved in energy production (NADH dehydrogenase subunit 5 and 6, BMC12393, BMC5921) and $\beta$-oxidation of fatty acid oxidation (3-ketoacyl-CoA thiolase, BMC07758) were highly expressed in male worms. Enzymes involved in glycolysis and the citric acid cycle were also male up-regulated in C. elegans and $T$. vitrinus $[3,18]$. These findings may reflect the high energy required for sperm motility, since fatty acids provide an efficient energy source. In addition to these enzymes, two Brugia clusters with high male expression ratios were homologous to C. elegans trehalases. The disaccharide trehalose has been proposed to have essential physiological functions in nematodes as a source of energy and as a protectant against environmental stress [44]. Increased trehalose transcription may reflect an increased energy requirement in male worms or their sperm.

\subsection{Gene expression in females}

Thirty-nine percent (255/650) of female up-regulated $B$. malayi clusters were novel. Forty-seven percent (303/650) of the female up-regulated clusters had significant similarity to genes from $C$. elegans. Many of these $C$. elegans genes were highly expressed in embryos by microarray experi- ments [4], and 47\% (112/237) of those studied by RNAi had phenotypes such as embryo lethality, growth defects and post-embryonic defects. Many of the annotated female upregulated clusters in our study were associated with embryonic development (fatty acid binding protein, chaperonin-like protein), eggshell formation (microfilaria sheath protein), factors involved in transcription and translation (RNA polymerase, tRNA-synthetase, ribosomal proteins), and energy supply (ATP synthase and enzymes in the citric acid cycle).

A few of these genes deserve more comment. A gene coding for a lipid-binding protein $(B m-F A B-1)$, has been previously shown to have female up-regulated expression [11,12]. Its localization in perivitelline fluid of developing embryos within the uteri of adult female worms supports the hypothesis that this protein is involved in transfer of fatty acids to developing embryos [45]. The gene coding for microfilaria sheath protein (Bm-SHP-1), an abundant eggshell protein, has previously been shown to be female up-regulated in filarial parasites [12], and the inactivation of MF sheath protein by RNAi in adult female $B$. malayi has been reported to inhibit MF release from female worms [13]. Chaperonins such as heat shock protein 70 (Bm-HSP-70) have been reported to have female up-regulated expression in other nematodes [18]. Chaperonins are large, multi-subunit proteins that facilitate the protein-folding process by providing a protected environment. Recent studies show that these molecules play a role in transduction and regulation of cGMP levels [46], and that they are essential for embryonic development in C. elegans $[47,48]$. The notable female up-regulated expression of a heat shock gene (Bm-shp-70) and chaperonins in our study suggests that these molecules may play a parallel role in B. malayi reproduction and perhaps also in protein synthesis. The fact that other genes required for protein synthesis had female upregulated expression is consistent with a high requirement for protein synthesis for egg production and larval development in female worms.

Several clusters with significant similarity to genes encoding histones 2 and 4 were expressed in a female up-regulated manner in B. malayi. Histones comprise a family of closely related, basic DNA-binding proteins that play a role in gene regulation. Several histones and histone-like proteins are differentially expressed between males and hermaphrodites in C. elegans [20]. RNAi of his-71, a histone-like gene with a female-enriched homologue in T. vitrinus, resulted in embryonic lethality in C. elegans [24,25].

Interestingly, genes coding for two $B$. malayi serine proteinase inhibitors (Bm-spn-1 and Bm-spn-2) had female up-regulated expression by microarray. $B m$-spn- 1 has been reported to be enriched in filarial L3 [49,50], and Bm-spn2 has been reported to be expressed only by microfilariae [51]. Increased expression of Bm-spn-2 in female worms suggests that this gene is also expressed in developing larvae and microfilariae within female worms. Bm-spn-2 has been suggested to have a role in immune evasion in filarial parasites [52]. Related mammalian proteins have been reported to be involved in remodeling of germ line tissues and 
migration of germ cells [53]. Additional studies are needed to establish whether these proteins are necessary for filarial reproduction.

Several clusters with significant similarity to transcription regulatory elements, (including high motility group proteins and Y-box factor), had female up-regulated expression, while clusters encoding transcription factors such as zinc finger proteins (BMC07636, BMC06355) were up-regulated in male worms. These elements may be involved in coordinated expression of gender-regulated transcripts in Brugia.

In conclusion, microarray analysis has provided the first broad view of gender-regulated gene expression in B. malayi. It is likely that many of these genes are involved in reproduction (gamete production, gamete function, and embryo development). Much more work will be required to understand how the products of these genes function and interact and to explore their potential as new drug targets. However, we believe this work represents an important step toward a deeper understanding of reproduction in filarial worms. More generally, this approach holds great promise as a means of studying stage-specific or tissue-specific gene expression in parasitic nematodes.

\section{Acknowledgments}

Steven Williams is Director of Molecular Resources, Filariasis Research Reagent Repository Center (founded by RFP-contract NIH-NIAID-DMID-03-24). The Brugia malayi Microarray Consortium worked together to plan and finance construction of the array. The authors wish to thank John D. McPherson, Ph.D. for the design of the oligonucleotides used on the microarray slides. In addition, we thank Michael Heinz for his excellent work in performing the hybridizations to the microarrays.

\section{Appendix A. Supplementary data}

Supplementary data associated with this article can be found, in the online version, at doi:10.1016/j.molbiopara. 2005.05.005.

\section{References}

[1] Boag PR, Gasser RB, Nisbet AJ, Newton SE. Genomics of reproduction in parasitic nematodes-fundamental and biotechnological implications. Biotechnol Adv 2001;21:103-8.

[2] Stothard P, Pilgrim D. Sex-determination gene and pathway evolution in nematodes. Bioessays 2003;25:221-31.

[3] Reinke V, Smith HE, Nance J, Wang J. A global profile of germline gene expression in C. elegans. Mol Cell 2000;6:605-16.

[4] Piano F, Schetter AJ, Morton DG, et al. Gene clustering based on RNAi phenotypes of ovary-enriched genes in C. elegans. Curr Biol 2002;12:1959-64.

[5] Blaxter ML, Ley PD, Garey JR, et al. A molecular evolutionary framework for the phylum Nematoda. Nature 1998;392:71-5.
[6] Burglin TR, Lobos E, Blaxter ML. Caenorhabditis elegans as a model for parasitic nematodes. Int J Parasitol 1998;28:395-411.

[7] Parkinson J, Whitton C, Schmid R, Thomson M, Blaxter ML. NEMBASE: a resource for parasitic nematode EST's. Nucleic Acids Res 2004;32:D427-30.

[8] Williams SA, Laney SJ. Filarial genomics: gene discovery and gene expression. In: Klei TR, Rajan TV, editors. World class parasites: the filaria, vol. 5. Boston MA: Kluwer Academic Publishers; 2003. p. 31-42.

[9] Ghedin E, Wang S, Foster JM, et al. First sequenced genome of a parasitic nematode. Trends Parasitol 2004;20:151-3.

[10] Kron M, Petridis M, Milev Y, et al. Expression, localization and alternative function of cytoplasmic asparaginyl-tRNA synthetase in Brugia malayi. Mol Biochem Parasitol 2003;129:33-9.

[11] Michalski ML, Weil GJ. Gender-specific gene expression in Brugia malayi. Mol Biochem Parasitol 1999;104:247-57.

[12] Li BW, Rush AC, Tan J, Weil GJ. Quantitative analysis of genderregulated transcripts in the filarial nematode Brugia malayi by realtime RT-PCR. Mol Biochem Parasitol 2004;137:329-37.

[13] Aboobaker AA, Blaxter ML. Use of RNA interference to investigate gene function in the human filarial nematode parasite Brugia malayi. Mol Biochem Parasitol 2003;129:41-5.

[14] Schena M, Shalon D, Davis RW, Brown PO. Quantitative monitoring of gene expression patterns with a complementary DNA microarray. Science 1995;270:368-9.

[15] Shalon D, Smith SJ, Brown PO. A DNA microarray system for analyzing complex samples using two-color fluorescent probe hybridization. Genome Res 1996;6:639-45.

[16] Blader IJ, Manger ID, Boothroyd JC. Microarray analysis reveals previously unkown changes in Toxoplasma gondii-infected human cells. J Biol Chem 2001;276:24223-31.

[17] Rathod PK, Ganesan K, Hayward RE, Bozdech Z, DeRisi JL. DNA microarray for malaria. Trends Parasitol 2002;18:39-45.

[18] Nisbet AJ, Gasser RB. Profiling of gender-specific gene expression for Trichostrongylus vitrinus (Nematoda: Strongylida) by microarray analysis of expressed sequence tag libraries constructed by suppressive-subtractive hybridisation. Int J Parasitol 2004;34:633-43.

[19] Akopyants NS, Matlib RS, Bukanova EN, et al. Expression profiling using random genomic DNA microarrays identifies differentially expressed genes associated with three major developmental stages of the protozoan parasite Leishmania major. Mol Biochem Parasitol 2004;136:71-86.

[20] Altschul SF, Gish W, Miller W, Myers EW, Lipman DJ. Basic local alignment search tool. J Mol Biol 1990;215:403-10.

[21] Clevel WS, Devlin SJ. Locally-weighted regression: an approach to regression analysis by local fitting. J Am Statist Assoc 1988;83:596-610.

[22] Benson DA, Karsch-Mizrachi I, Lipman DJ, Ostell J, Wheeler DL. GenBank. Nucleic Acids Res 2003;31:23-7.

[23] Boguski MS, Lowe TM, Tolstoshev CM. dbEST-database for "expressed sequence tags". Nat Genet 1993;4:332-3.

[24] Kamath RS, Fraser AG, Dong Y, Poulin G, Durbin R, et al. Systematic functional analysis of the Caenorhabditis elegans genome using RNAi. Nature 2003;421:220-1.

[25] Maeda I, Kohara Y, Yamamoto M, Sugimoto A. Large-scale analysis of gene function in Caenorhabditis elegans by high-throughput RNAi. Curr Biol 2001;11:171-6.

[26] Edgar R, Domrachev M, Lash AE. Gene expression omnibus: NCBI gene expression and hybridization array data repostitory. Nucleic Acid Res 2002;30:207-10.

[27] Jiang M, Ryu J, Kiraly M, Duke K, Reinke V, Kim SK. Genomewide analysis of developmental and sex-regulated gene expression profiles in Caenorhabditis elegans. Proc Natl Acad Sci USA 2001;98:218-23.

[28] Boag PR, Newton SE, Gasser RB. Molecular aspects of sexual development and reproduction in nematodes and schistosomes. Adv Parasitol 2001;50:153-98. 
[29] Boag PR, Ren P, Newton SE, Gasser RB. Molecular characterization of a male-specific serine/threonine phosphatase from Oesophagostomum dentatum (Nematoda: Strongylida), and functional analysis of homologues in Caenorhabditis elegans. Int J Parasitol 2003;33:313-25.

[30] Ward S, Klass M. The localization of the major sperm protein in Caenorhhabditis elegans sperm and spermatocytes. Dev Biol 1982;92:203-8.

[31] Scott A, Dinman J, Sussman D, Yenbutr P, Ward S. Major sperm protein genes from Oncocerca volvulus. Mol Biochem Parasitol 1989;36:119-26.

[32] Robert TM, Stewart M. Acting like actin: the dynamics of the nematode major sperm protein (MSP) cytoskeleton indicate a push-pull mechanism for amoeboid cell motility. J Cell Biol 2000;149:7-12.

[33] Miller MA, Nguyue AQ, Lee M, et al. A sperm cytoskeletal protein that signals oocyte meiotic maturation and ovulation. Science 2001;291:2144-7.

[34] Miller M, Ruest PJ, Kosinski M, Hanks SK, Greenstein D. An Eph receptor sperm-sensing control mechanism for oocyte meiotic maturation in Caenorhabditis elegans. Genes Dev 2003;17:187-200.

[35] Hanazawa M, Mochii M, Ueno N, Kohara Y, Iino Y. Use of cDNA subtraction and RNA interference screens in combination reveals genes required for germ-line development in Caenorhabditis elegans. Proc Natl Acad Sci USA 2001;98:8686-91.

[36] Harris BZ, Lim WA. Mechanism and role of PDZ domains in signaling complex assembly. J Cell Sci 2001;114:3219-31.

[37] Cox DN, Chao A, Baker J, et al. A novel class of evolutionarily conserved genes defined by piwi are essential for stem cell selfrenewal. Genes Dev 1998;12:3715-27.

[38] Davis AH, Blanton R, Klich P. Stage and sex specific differences in actin gene expression in Schistosoma mansoni. Mol Biochem Parasitol 1985;17:289-98.

[39] Hoffmann KF, Johnston DA, W. Dunne D. Identification of Schistosoma mansoni gender-associated gene transcripts by cDNA microarray profiling. Genome Biol 2002;3:41.

[40] Vilfan A. The binding dynamics of tropomyosin on actin. Biophys J 2001;81:3146-55.

[41] Clough ER. Morphology and reproductive organs and oogenesis in bisexual and unisexual transplants of mature Schistosoma mansoni females. J Parasitol 1981;67:535-9.
[42] Oberlander U, Adam R, Berg K, Seeber F, Lucius R. Molecular cloning and characterization of the filarial LIM domain proteins AvL3-1 and OvL3-1. Exp Parasitol 1995;81:592-9.

[43] Seeber F, Brattig N, Soboslay PT, et al. Characterization of a recombinant T cell and B cell reactive polypeptide of Onchocerca volvulus. J Immunol 1993;150:2931-44.

[44] Pellerone FI, Archer SK, Behm CA, Grant WN, Lacey MJ, Somerville AC. Trehalose metabolism genes in Caenorhabditis elegans and filarial nematodes. Int J Parasitol 2003;33:1195-206.

[45] Michalski ML, Monsey JD, Cistola DP, Weil GJ. An embryoassociated fatty acid-binding protein in the filarial nematode Brugia malayi. Mol Biochem Parasitol 2002;124:1-10.

[46] Brinby DA, Link EM, Vowels JJ, Tian H, Colacurcio PL, Thomas JH. A transmembrane guanylyl cyclase (DAF-11) and Hsp90 (DAF-21) regulate a common set of chemosensory behaviors in Caenorhabditis elegans. Genetics 2002;155:85-104.

[47] Linder B, Jin Z, Freedman JH, Rubin CS. Molecular characterization of a novel, developmentally regulated small embryonic chaperone from Caenorhabditis elegans. J Biol Chem 1996;271:3015866.

[48] Walhout A, Reboul J, Shtanko O, et al. Integrating interactome, phenome, and transcriptome mapping data for the $C$. elegans germline. Curr Biol 2002;12:1952-8.

[49] Pastrana DV, Raghavan N, FitzGerald P, et al. Filarial nematode parasites secrete a homologue of the human cytokine macrophage migration inhibitory factor. Infect Immun 1998;66:5955-63.

[50] Allen JE, Daub J, Guiliano D, et al. Analysis of genes expressed at the infective larval stage validates utility of Litomosoides sigmodontis as a murine model for filarial vaccine development. Infect Immun 2000;68:545-8.

[51] Zang X, Atmadja AK, Gray $P$, et al. The serpin secreted by Brugia malayi microfilariae, Bm-SPN-2, elicits strong, but short-lived, immune responses in mice and humans. J Immunol 2000;165:5161-9.

[52] Maizels RM, Gomez-Escobar N, Gregory WF, Murray J, Zang $\mathrm{X}$. Immune evasion genes from filarial nematodes. Int $\mathrm{J}$ Parasitol 2001;31:889-98.

[53] Monsees TK, Schill WB, Miska W. Protease-protease inhibitor interactions in Sertoli cell-germ cell crosstalk. Adv Exp Med Biol 1997;424:111-23 